\title{
Exact controllability for a wave equation with fixed boundary control
}

Lizhi Cui $^{1,2^{*}}$ and Libo Song ${ }^{3}$

\section{"Correspondence:}

cuilz829@nenu.edu.cn

${ }^{1}$ College of Applied Mathematics,

Jilin University of Finance and

Economics, Changchun, 130117,

China

${ }^{2}$ School of Mathematics and

Statistics, Northeast Normal

University, Changchun, 130024,

China

Full list of author information is

available at the end of the article

\begin{abstract}
This paper addresses the study of the controllability for a one-dimensional wave equation in domains with moving boundary. This equation characterizes the motion of a string with a fixed endpoint and the other one moving. When the speed of the moving endpoint is less than $1-\frac{2}{1+e^{2}}$, by the Hilbert Uniqueness Method, the exact controllability of this equation is established. Also, the explicit dependence of the controllability time on the speed of the moving endpoint is given.
\end{abstract}

Keywords: exact controllability; non-cylindrical domain; wave equation

\section{Introduction}

Given $T>0$. Let us consider the non-cylindrical domain $\widehat{Q}_{T}^{k}$, defined by

$$
\widehat{Q}_{T}^{k}=\left\{(y, t) \in \mathbb{R}^{2} ; 0<y<\alpha_{k}(t) \text {, for all } t \in(0, T)\right\}
$$

where

$$
\alpha_{k}(t)=1+k t, \quad 0<k<1 .
$$

Consider the following controlled wave equation in the non-cylindrical domain $\widehat{Q}_{T}^{k}$ :

$$
\left\{\begin{array}{lll}
u_{t t}-u_{y y}=0 & & \text { in } \widehat{Q}_{T}^{k}, \\
u(0, t)=v(t), & u\left(\alpha_{k}(t), t\right)=0 & \text { on }(0, T), \\
u(0)=u^{0}, & u_{t}(0)=u^{1} & \text { in }(0,1),
\end{array}\right.
$$

where $u$ is the state variable, $v$ is the control variable and $\left(u^{0}, u^{1}\right) \in L^{2}(0,1) \times H^{-1}(0,1)$ is any given initial value. Equation (1.2) may describe the motion of a string with a fixed endpoint and a moving one. The constant $k$ is called the speed of the moving endpoint. By [1], for $0<k<1$, any $\left(u^{0}, u^{1}\right) \in L^{2}(0,1) \times H^{-1}(0,1)$ and $v \in L^{2}(0, T)$, (1.2) admits a unique solution in the sense of a transposition.

The main purpose of this paper is to study the exact controllability of (1.2). As we all know, there exists much literature on the controllability problems of wave equations in a cylindrical domain. However, there are only a few works on the exact controllability for wave equations defined in non-cylindrical domains. We refer to [1-3], and [4] for some known results in this respect. In [2], the exact controllability of a multi-dimensional wave

\section{是 Springer}

(O2014 Cui and Song; licensee Springer. This is an Open Access article distributed under the terms of the Creative Commons Attribution License (http://creativecommons.org/licenses/by/2.0), which permits unrestricted use, distribution, and reproduction in any medium, provided the original work is properly cited. 
equation with constant coefficients in a non-cylindrical domain was established, while the control entered the system through the whole non-cylindrical domain. In [1] and [3], some controllability results for the wave equations with Dirichlet boundary conditions in suitable non-cylindrical domains were investigated, respectively. But some additional conditions on the moving boundary were required, which entail the method used in [1] and [3] not to be applicable to the controllability problems of (1.2). In [1] and [3] in the one-dimensional case, the following condition seems necessary:

$$
\int_{0}^{\infty}\left|\alpha_{k}^{\prime}(t)\right| d t<\infty
$$

It is easy to check that this condition is not satisfied for the moving boundary in (1.2). The control system of this paper is similar to that of [4]. But the control is put on a different boundary. We mainly use the multiplier method to overcome these difficulties and drop the additional conditions for the moving boundary. But the simple multiplier in [4] is not applicable to the controllability problem of (1.2). We choose the complicated multiplier which satisfies the first-order linear differential equation. But the result in this paper is not satisfactory. We hope that the controllability result is obtained when $k \in(0,1)$. We hope that we obtain a modified multiplier in the forthcoming papers.

The rest of this paper is organized as follows. In Section 2, we give some preliminaries and the main results. In Section 3, we prove that the Hilbert Uniqueness Method (HUM) works very well for (1.2). Section 4 contains the proofs of the important inequalities used in Section 3.

\section{Preliminaries and main results}

The goal of this paper is to study the exact controllability of (1.2) in the following sense.

Definition 2.1 Equation (1.2) is called exactly controllable at the time $T$, if for any initial value $\left(u^{0}, u^{1}\right) \in L^{2}(0,1) \times H^{-1}(0,1)$ and any target $\left(u_{d}^{0}, u_{d}^{1}\right) \in L^{2}\left(0, \alpha_{k}(T)\right) \times H^{-1}\left(0, \alpha_{k}(T)\right)$, one can always find a control $v \in L^{2}(0, T)$ such that the corresponding solution $u$ of (1.2) in the sense of a transposition satisfies

$$
u(T)=u_{d}^{0} \quad \text { and } \quad u_{t}(T)=u_{d}^{1}
$$

Denote $T_{k}^{*}$ for a controllability time. The main result of this paper is stated as follows.

Theorem 2.1 Suppose that $0<k<1-\frac{2}{1+e^{2}}$. For any given $T>T_{k}^{*},(1.2)$ is exactly controllable at time $T$ in the sense of Definition 2.1 .

Remark 2.1 It seems natural to expect that the exact controllability of (1.2) holds when $k \in(0,1)$. However, we did not have success in extending the approach developed in Theorem 2.1 to this case.

In order to prove Theorem 2.1, we first transform (1.2) into a wave equation with variable coefficients in a cylindrical domain. To this aim, set

$$
x=\frac{y}{\alpha_{k}(t)} \quad \text { and } \quad w(x, t)=u(y, t)=u\left(\alpha_{k}(t) x, t\right) \quad \text { for }(y, t) \in \widehat{Q}_{T}^{k} .
$$


Then, it is easy to check that $(x, t)$ varies in $Q:=(0,1) \times(0, T)$. Also, $(1.2)$ is transformed into the following equivalent wave equation in the cylindrical domain $Q$ :

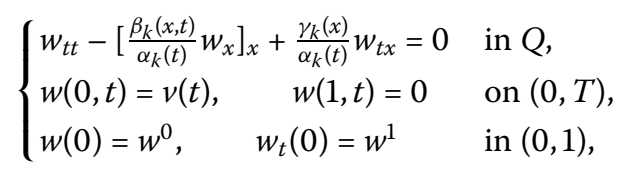

where

$$
\beta_{k}(x, t)=\frac{1-k^{2} x^{2}}{\alpha_{k}(t)}, \quad \gamma_{k}(x)=-2 k x, \quad w^{0}=u^{0}, \quad w^{1}=u^{1}+k x u_{x}^{0} .
$$

Equation (2.1) admits a unique solution in the sense of a transposition,

$$
w \in C\left([0, T] ; L^{2}(0,1)\right) \cap C^{1}\left([0, T] ; H^{-1}(0,1)\right)
$$

(see [5]).

Therefore, the exact controllability of (1.2) (Theorem 2.1) is reduced to the following main controllability result for the wave equation (2.1).

Theorem 2.2 Suppose that $0<k<1-\frac{2}{1+e^{2}}$. Let $T>T_{k}^{*}$. Then, for any initial value $\left(w^{0}, w^{1}\right) \in L^{2}(0,1) \times H^{-1}(0,1)$ and target $\left(w_{d}^{0}, w_{d}^{1}\right) \in L^{2}(0,1) \times H^{-1}(0,1)$, there exists a control $v \in L^{2}(0, T)$ such that the corresponding solution $w$ of (2.1) in the sense of transposition satisfies

$$
w(T)=w_{d}^{0} \quad \text { and } \quad w_{t}(T)=w_{d}^{1} .
$$

The key proof of Theorem 2.2 is to prove two important inequalities for the following homogeneous wave equation in cylindrical domains. We have

$$
\begin{cases}\alpha_{k}(t) z_{t t}-\left[\beta_{k}(x, t) z_{x}\right]_{x}+\gamma_{k}(x) z_{t x}=0 & \text { in } Q, \\ z(0, t)=0, \quad z(1, t)=0 & \text { on }(0, T), \\ z(0)=z^{0}, \quad z_{t}(0)=z^{1} & \text { in }(0,1),\end{cases}
$$

where $k \in(0,1),\left(z^{0}, z^{1}\right) \in H_{0}^{1}(0,1) \times L^{2}(0,1)$ is any given initial value, and $\alpha_{k}, \beta_{k}$, and $\gamma_{k}$ are the functions given in (2.1). Similar to Theorem 3.2 in [5], we see that (2.3) has a unique weak solution

$$
z \in C\left([0, T] ; H_{0}^{1}(0,1)\right) \cap C^{1}\left([0, T] ; L^{2}(0,1)\right) .
$$

Define the following weighted energy for (2.3):

$$
E(t)=\frac{1}{2} \int_{0}^{1}\left[\alpha_{k}(t)\left|z_{t}(x, t)\right|^{2}+\beta_{k}(x, t)\left|z_{x}(x, t)\right|^{2}\right] d x \quad \text { for } t \geq 0,
$$

where $z$ is the solution of (2.3). It follows that

$$
E_{0} \triangleq E(0)=\frac{1}{2} \int_{0}^{1}\left[\left|z^{1}(x)\right|^{2}+\beta_{k}(x, 0)\left|z_{x}^{0}(x)\right|^{2}\right] d x .
$$


In the sequel, we denote by $C$ a positive constant depending only on $T$ and $k$, which may be different from one place to another.

We obtain the following two lemmas whose proof are found in [4].

Lemma 2.1 For any $\left(z^{0}, z^{1}\right) \in H_{0}^{1}(0,1) \times L^{2}(0,1)$ and $t \in[0, T]$, we have

$$
E(t)=\frac{1}{\alpha_{k}(t)} E_{0}
$$

Lemma 2.2 Suppose that $q \in C^{1}([0,1])$ is any given function. Then any solution $z$ of (2.3) satisfies the following estimate:

$$
\begin{aligned}
& {\left.\left[\frac{1}{2} \int_{0}^{T} \beta_{k}(x, t) q(x)\left|z_{x}(x, t)\right|^{2} d t\right]\right|_{0} ^{1}} \\
& =\frac{1}{2} \int_{0}^{T} \int_{0}^{1}\left[q_{x}(x) \alpha_{k}(t)\left|z_{t}(x, t)\right|^{2}+\left(q_{x}(x) \beta_{k}(x, t)-\beta_{k, x}(x, t) q(x)\right)\left|z_{x}(x, t)\right|^{2}\right] d x d t \\
& \quad-\int_{0}^{T} \int_{0}^{1} \alpha_{k, t}(t) q(x) z_{t}(x, t) z_{x}(x, t) d x d t \\
& \quad+\left.\int_{0}^{1}\left[\alpha_{k}(t) q(x) z_{t}(x, t) z_{x}(x, t)+\frac{1}{2} \gamma_{k}(x) q(x)\left|z_{x}(x, t)\right|^{2}\right] d x\right|_{0} ^{T} .
\end{aligned}
$$

In order to prove Theorem 2.2, we need the following two important inequalities. The proofs of two important inequalities are given in Section 4.

Theorem 2.3 Let $T>0$. For any $\left(z^{0}, z^{1}\right) \in H_{0}^{1}(0,1) \times L^{2}(0,1)$, there exists a constant $C>0$ such that the corresponding solution $z$ of (2.3) satisfies

$$
\int_{0}^{T} \beta_{k}(0, t)\left|z_{x}(0, t)\right|^{2} d t \leq C\left(\left|z^{0}\right|_{H_{0}^{1}(0,1)}^{2}+\left|z^{1}\right|_{L^{2}(0,1)}^{2}\right)
$$

Theorem 2.4 Let $T>T_{k}^{*}$. For any $\left(z^{0}, z^{1}\right) \in H_{0}^{1}(0,1) \times L^{2}(0,1)$, there exists a constant $C>0$ such that the corresponding solution $z$ of (2.3) satisfies

$$
\int_{0}^{T} \beta_{k}(0, t)\left|z_{x}(0, t)\right|^{2} d t \geq C\left(\left|z^{0}\right|_{H_{0}^{1}(0,1)}^{2}+\left|z^{1}\right|_{L^{2}(0,1)}^{2}\right)
$$

\section{Application of HUM}

In this section, we prove the exact controllability for the wave equation (2.1) in the cylindrical domain $Q$ (Theorem 2.2) by HUM.

Proof of Theorem 2.2 We divide the proof of Theorem 2.2 into three parts. We use certain inequalities proved later in Section 4.

Step 1. First, we define a linear operator $\Lambda: H_{0}^{1}(0,1) \times L^{2}(0,1) \rightarrow H^{-1}(0,1) \times L^{2}(0,1)$.

For any $\left(z^{0}, z^{1}\right) \in H_{0}^{1}(0,1) \times L^{2}(0,1)$, denote by $z$ the corresponding solution of (2.3). Consider the following homogeneous wave equation:

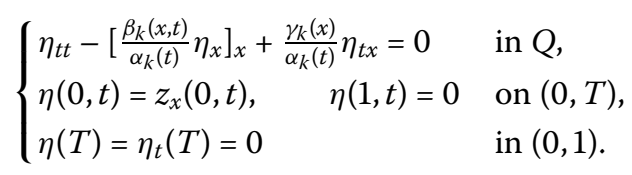


Then it is well known that (3.1) admits a unique solution in the sense of a transposition,

$$
\eta \in C\left([0, T] ; L^{2}(0,1)\right) \cap C^{1}\left([0, T] ; H^{-1}(0,1)\right) .
$$

Moreover, by Theorem 2.3 in Section 3, there exists a constant $C$ such that

$$
|\eta|_{C\left([0, T] ; L^{2}(0,1)\right) \cap C^{1}\left([0, T] ; H^{-1}(0,1)\right)} \leq C\left|z_{x}(0, \cdot)\right|_{L^{2}(0, T)} \leq C\left(\left|z^{0}\right|_{H_{0}^{1}(0,1)}+\left|z^{1}\right|_{L^{2}(0,1)}\right) .
$$

Define a linear operator $\Lambda$ :

$$
\begin{aligned}
& \Lambda: H_{0}^{1}(0,1) \times L^{2}(0,1) \rightarrow H^{-1}(0,1) \times L^{2}(0,1), \\
& \left(z^{0}, z^{1}\right) \mapsto\left(-\eta_{t}(\cdot, 0)-\gamma_{k}(\cdot) \eta_{x}(\cdot, 0)+k \eta(\cdot, 0), \eta(\cdot, 0)\right),
\end{aligned}
$$

where we use $z$ to denote the solution of (2.3) associated to $z^{0}$ and $z^{1}$, and $\eta$ denotes the solution of and (3.1) associated to $z$.

Step 2. That $\Lambda$ is an isomorphism is equivalent to the exact controllability of (2.1). In fact, for any target $\left(w_{d}^{0}, w_{d}^{1}\right) \in L^{2}(0,1) \times H^{-1}(0,1)$, the following wave equation:

$$
\left\{\begin{array}{lll}
\xi_{t t}-\left[\frac{\beta_{k}(x, t)}{\alpha_{k}(t)} \xi_{x}\right]_{x}+\frac{\gamma_{k}(x)}{\alpha_{k}(t)} \xi_{t x}=0 & \text { in } Q, \\
\xi(0, t)=0, & \xi(1, t)=0 & \text { on }(0, T), \\
\xi(T)=w_{d}^{0}, & \xi_{t}(T)=w_{d}^{1} & \text { in }(0,1)
\end{array}\right.
$$

has a unique solution $\xi \in C\left([0, T] ; L^{2}(0,1)\right) \cap C^{1}\left([0, T] ; H^{-1}(0,1)\right)$.

Suppose that $\Lambda$ is an isomorphism, for any initial value $\left(w^{0}, w^{1}\right) \in L^{2}(0,1) \times H^{-1}(0,1)$, there exists $\left(z^{0}, z^{1}\right) \in H_{0}^{1}(0,1) \times L^{2}(0,1)$ such that

$$
\Lambda\left(z^{0}, z^{1}\right)=\left(-\left[w^{1}-\xi_{t}(0)\right]+k\left[w^{0}-\xi(0)\right]-\gamma_{k}\left[w_{x}^{0}-\xi_{x}(0)\right],\left[w^{0}-\xi(0)\right]\right) .
$$

Note that $\eta$ is the solution of (3.1) and that $z$ is the solution of (2.3) associated to $\left(z^{0}, z^{1}\right)$. Then, by the definition of $\Lambda$, we have

$$
\Lambda\left(z^{0}, z^{1}\right)=\left(-\eta_{t}(\cdot, 0)-\gamma_{k}(\cdot) \eta_{x}(\cdot, 0)+k \eta(\cdot, 0), \eta(\cdot, 0)\right) .
$$

By (3.4) and (3.5), it follows that $\left(\eta(0), \eta_{t}(0)\right)=\left(w^{0}-\xi(0), w^{1}-\xi_{t}(0)\right)$. If we set $w=\xi+\eta$, by the uniqueness of (3.3), then $w$ is the solution of (2.1) associated to $v=z_{x}(0, \cdot)$. Furthermore, $\left(w(0), w_{t}(0)\right)=\left(w^{0}, w^{1}\right)$ and $\left(w(T), w_{t}(T)\right)=\left(w_{d}^{0}, w_{d}^{1}\right)$. Therefore, we get the exact controllability of (2.1).

Step 3. Now we prove that $\Lambda$ is an isomorphism, when $T>T_{k}^{*}$.

Write $F=H_{0}^{1}(0,1) \times L^{2}(0,1)$ and denote by $F^{\prime}$ its conjugate space. Also, define a bilinear form $A$ on $\left(H_{0}^{1}(0,1) \times L^{2}(0,1)\right)^{2}$ as follows:

$$
\begin{aligned}
& A\left(\left(z^{0}, z^{1}\right),\left(y^{0}, y^{1}\right)\right) \\
& \quad \triangleq\left\langle\Lambda\left(z^{0}, z^{1}\right),\left(y^{0}, y^{1}\right)\right\rangle_{F^{\prime}, F} \\
& \quad=\left\langle-\eta_{t}(\cdot, 0)-\gamma_{k}(\cdot) \eta_{x}(\cdot, 0), y^{0}\right\rangle_{H^{-1}, H_{0}^{1}}+\int_{0}^{1}\left[k \eta(x, 0) y^{0}(x)+\eta(x, 0) y^{1}(x)\right] d x,
\end{aligned}
$$

for any $\left(z^{0}, z^{1}\right),\left(y^{0}, y^{1}\right) \in H_{0}^{1}(0,1) \times L^{2}(0,1)$, where $\eta$ denotes the solution of (3.1). 
Multiplying the first equation of (3.1) by $\alpha_{k}(t) z$ and integrating on $Q$, by (2.3), we obtain

$$
\begin{aligned}
& \int_{0}^{T} \beta_{k}(0, t)\left|z_{x}(0, t)\right|^{2} d t \\
& \quad=\left\langle-\eta_{t}(\cdot, 0)-\gamma_{k}(\cdot) \eta_{x}(\cdot, 0), z^{0}\right\rangle_{H^{-1}, H_{0}^{1}}+\int_{0}^{1}\left[k \eta(x, 0) z^{0}(x)+\eta(x, 0) z^{1}(x)\right] d x .
\end{aligned}
$$

Combining the above equality with the definition of $\Lambda$, we have

$$
\int_{0}^{T} \beta_{k}(0, t)\left|z_{x}(0, t)\right|^{2} d t=\left\langle\Lambda\left(z^{0}, z^{1}\right),\left(z^{0}, z^{1}\right)\right\rangle_{F^{\prime}, F}
$$

By Theorem 2.3 and Theorem 2.4, it suffices to prove that $\Lambda$ is surjective. Notice that Theorem 2.4 and (3.6) imply $A$ is a coercive bilinear form. Moreover, by (3.2), it is easy to check that $A$ is bounded. Therefore, by the Lax-Milgram Theorem, $\Lambda$ is a surjection. It follows that $\Lambda$ is an isomorphism.

Remark 3.1 By the equivalent transformation in Section 2, Theorem 2.2 implies the exact controllability for in the non-cylindrical domain $\widehat{Q}_{T}^{k}$ at the time $T>T_{k}^{*}$ (Theorem 2.1).

\section{The proofs of important inequalities}

In this section, we give proofs of Theorem 2.3 and Theorem 2.4.

Proof of Theorem 2.3 First, we choose $q(x)=x-1$ for $x \in[0,1]$ in (2.5). Noting that $\alpha_{k}^{\prime}(t)=$ $k, \beta_{k, x}(x, t)=\frac{-2 k^{2} x}{1+k t}$ and $\gamma_{k}(x)=-2 k x$, it follows that

$$
\begin{aligned}
& \frac{1}{2} \int_{0}^{T} \beta_{k}(0, t)\left|z_{x}(0, t)\right|^{2} d t \\
& \quad=\int_{0}^{T} E(t) d t-\int_{0}^{T} \int_{0}^{1} k(x-1) z_{t}(x, t) z_{x}(x, t) d x d t \\
& \quad+\int_{0}^{T} \int_{0}^{1} \frac{k^{2} x(x-1)}{1+k t}\left|z_{x}(x, t)\right|^{2} d x d t \\
& \quad+\left.\int_{0}^{1}\left[\alpha_{k}(t)(x-1) z_{t}(x, t) z_{x}(x, t)-k x(x-1)\left|z_{x}(x, t)\right|^{2}\right] d x\right|_{0} ^{T} .
\end{aligned}
$$

Next, we estimate every terms on the right side of (4.1). Notice that $1 \leq \alpha_{k}(t) \leq 1+k T$ and $0<\frac{1-k^{2}}{1+k T} \leq \beta_{k}(x, t) \leq 1$ for any $(x, t) \in Q$. By (2.4), we have

$$
\begin{aligned}
& \int_{0}^{T} E(t) d t-\int_{0}^{T} \int_{0}^{1} k(x-1) z_{t}(x, t) z_{x}(x, t) d x d t+\int_{0}^{T} \int_{0}^{1} \frac{k^{2} x(x-1)}{1+k t}\left|z_{x}(x, t)\right|^{2} d x d t \\
& \quad \leq \int_{0}^{T} E(t) d t+C \int_{0}^{T} \int_{0}^{1}\left[\left|z_{t}(x, t)\right|^{2}+\left|z_{x}(x, t)\right|^{2}\right] d x d t \\
& \quad \leq \int_{0}^{T} E(t) d t+C \int_{0}^{T} \int_{0}^{1}\left[\alpha_{t}(t)\left|z_{t}(x, t)\right|^{2}+\beta_{k}(x, t)\left|z_{x}(x, t)\right|^{2}\right] d x d t \\
& \quad \leq C \int_{0}^{T} E(t) d t \leq C E_{0} .
\end{aligned}
$$


On the other hand, for each $t \in[0, T]$, we have

$$
\begin{aligned}
& \left|\int_{0}^{1}\left[\alpha_{k}(t)(x-1) z_{t}(x, t) z_{x}(x, t)-k x(x-1)\left|z_{x}(x, t)\right|^{2}\right] d x\right| \\
& \quad \leq C E(t) \leq C E_{0} .
\end{aligned}
$$

Therefore, by (4.1)-(4.3), we have

$$
\frac{1}{2} \int_{0}^{T} \beta_{k}(0, t)\left|z_{x}(0, t)\right|^{2} d t \leq C E_{0} \leq C\left(\left|z^{0}\right|_{H_{0}^{1}(0,1)}^{2}+\left|z^{1}\right|_{L^{2}(0,1)}^{2}\right) .
$$

Remark 4.1 Theorem 2.3 implies that for any $\left(z_{0}, z_{1}\right) \in H_{0}^{1}(0,1) \times L^{2}(0,1)$, the corresponding solution $z$ of $(2.3)$ satisfies $z_{x}(0, \cdot) \in L^{2}(0, T)$.

In the following, we give a proof of Theorem 2.4.

Proof of Theorem 2.4 First, let $q$ be the solution of the following problem:

$$
\left\{\begin{array}{l}
q^{\prime}(x)=1+\frac{\beta_{k, x}(x, t)}{\beta_{k}(x, t)} q(x), \quad x \in[0,1) \\
q(1)=0
\end{array}\right.
$$

e.g.,

$$
q(x)=\frac{1-k^{2} x^{2}}{2 k}\left[\ln \frac{1+k x}{1-k x}-\ln \frac{1+k}{1-k}\right] .
$$

It is easy to check that

$$
\begin{aligned}
& q(x) \leq 0, \quad x \in[0,1] ; \\
& q^{\prime}(x) \geq 1, \quad x \in[0,1] ; \\
& q^{\prime}(x) \beta_{k}(x, t)-\beta_{k, x}(x, t) q(x)=\beta_{k}(x, t) ; \\
& M \triangleq \max _{0 \leq x \leq 1}|q(x)|=|q(0)|=\frac{1}{2 k} \ln \frac{1+k}{1-k} .
\end{aligned}
$$

Set

$$
g(x)=\frac{k^{2}}{1-k^{2} x^{2}} q^{2}(x), \quad x \in[0,1],
$$

then it is easy to check that

$$
M_{1} \triangleq \max _{0 \leq x \leq 1}|g(x)|=k^{2} q^{2}(0)=k^{2} M^{2}
$$

By (2.5) and (4.5), after calculating, we have

$$
\begin{aligned}
- & \frac{1}{2} q(0) \int_{0}^{T} \beta_{k}(0, t)\left|z_{x}(0, t)\right|^{2} d t \\
\quad= & \frac{1}{2} \int_{0}^{T} \int_{0}^{1}\left[q_{x}(x) \alpha_{k}(t)\left|z_{t}(x, t)\right|^{2}+\beta_{k}(x, t)\left|z_{x}(x, t)\right|^{2}\right] d x d t
\end{aligned}
$$




$$
\begin{aligned}
& -\int_{0}^{T} \int_{0}^{1} \alpha_{k, t}(t) q(x) z_{t}(x, t) z_{x}(x, t) d x d t \\
& +\left.\int_{0}^{1}\left[\alpha_{k}(t) q(x) z_{t}(x, t) z_{x}(x, t)+\frac{1}{2} \gamma_{k}(x) q(x)\left|z_{x}(x, t)\right|^{2}\right] d x\right|_{0} ^{T} \\
\geq & \frac{1}{2} \int_{0}^{T} \int_{0}^{1}\left[\alpha_{k}(t)\left|z_{t}(x, t)\right|^{2}+\beta_{k}(x, t)\left|z_{x}(x, t)\right|^{2}\right] d x d t \\
& -\int_{0}^{T} \int_{0}^{1} \alpha_{k, t}(t) q(x) z_{t}(x, t) z_{x}(x, t) d x d t \\
& +\left.\int_{0}^{1}\left[\alpha_{k}(t) q(x) z_{t}(x, t) z_{x}(x, t)+\frac{1}{2} \gamma_{k}(x) q(x)\left|z_{x}(x, t)\right|^{2}\right] d x\right|_{0} ^{T} .
\end{aligned}
$$

From (4.6), it follows that

$$
\begin{aligned}
& -\frac{1}{2} q(0) \int_{0}^{T} \beta_{k}(0, t)\left|z_{x}(0, t)\right|^{2} d t \\
& \quad \geq \int_{0}^{T} E(t) d t-\int_{0}^{T} \int_{0}^{1} k q(x) z_{t}(x, t) z_{x}(x, t) d x d t \\
& \quad+\left.\int_{0}^{1}\left[\alpha_{k}(t) q(x) z_{t}(x, t) z_{x}(x, t)+\frac{1}{2} \gamma_{k}(x) q(x)\left|z_{x}(x, t)\right|^{2}\right] d x\right|_{0} ^{T} .
\end{aligned}
$$

Next, we estimate the terms on the right side of (4.7). For each $t \in[0, T]$ and $\varepsilon>0$, we have

$$
\begin{aligned}
& \left|\int_{0}^{1}\left[\alpha_{k}(t) q(x) z_{t}(x, t) z_{x}(x, t)+\frac{1}{2} \gamma_{k}(x) q(x)\left|z_{x}(x, t)\right|^{2}\right] d x\right| \\
& \quad \leq\left|\int_{0}^{1} \alpha_{k}(t) q(x) z_{t}(x, t) z_{x}(x, t) d x\right|+\left.k\left|\int_{0}^{1} x q(x)\right| z_{x}(x, t)\right|^{2} d x \mid \\
& \quad \leq \sqrt{1+k t}\left[\frac{1}{2 \varepsilon} \int_{0}^{1} \alpha_{k}(t)\left|z_{t}(x, t)\right|^{2} d x+\frac{\varepsilon}{2} \int_{0}^{1} q^{2}(x)\left|z_{x}(x, t)\right|^{2} d x\right] \\
& \quad+k M \int_{0}^{1}\left|z_{x}(x, t)\right|^{2} d x \\
& \leq \frac{\sqrt{1+k t}}{2 \varepsilon} \int_{0}^{1} \alpha_{k}(t)\left|z_{t}(x, t)\right|^{2} d x+\frac{\sqrt{1+k t} \varepsilon M^{2}+2 k M}{2} \int_{0}^{1}\left|z_{x}(x, t)\right|^{2} d x \\
& \leq \frac{\sqrt{1+k t}}{\varepsilon} \frac{1}{2} \int_{0}^{1} \alpha_{k}(t)\left|z_{t}(x, t)\right|^{2} d x \\
& \quad+\frac{\left(\sqrt{1+k t} \varepsilon M^{2}+2 k M\right)(1+k t)}{1-k^{2}} \frac{1}{2} \int_{0}^{1} \beta_{k}(x, t)\left|z_{x}(x, t)\right|^{2} d x .
\end{aligned}
$$

Take $\varepsilon=\frac{1-k}{\sqrt{1+k t} M}$, then it is easy to check that

$$
\varepsilon>0 \quad \text { and } \quad \frac{\sqrt{1+k t}}{\varepsilon}=\frac{\left(\sqrt{1+k t} \varepsilon M^{2}+2 k M\right)(1+k t)}{1-k^{2}}=\frac{M(1+k t)}{1-k} .
$$

This implies that for any $t \in[0, T]$,

$$
\left|\int_{0}^{1}\left[\alpha_{k}(t) q(x) z_{t}(x, t) z_{x}(x, t)+\frac{1}{2} \gamma_{k}(x) q(x)\left|z_{x}(x, t)\right|^{2}\right] d x\right| \leq \frac{M(1+k t)}{1-k} E(t)=\frac{M}{1-k} E_{0} .
$$


It follows that

$$
\left|\int_{0}^{1}\left[\alpha_{k}(t) q(x) z_{t}(x, t) z_{x}(x, t)+\frac{1}{2} \gamma_{k}(x) q(x)\left|z_{x}(x, t)\right|^{2}\right] d x\right|_{0}^{T} \mid \leq \frac{2 M}{1-k} E_{0} .
$$

On the other hand, for any given $\varepsilon \in(0,1)$, we have

$$
\begin{aligned}
& \left|\int_{0}^{T} \int_{0}^{1} k q(x) z_{t}(x, t) z_{x}(x, t) d x d t\right| \\
& \quad=\left|\int_{0}^{T} \int_{0}^{1} \sqrt{1+k t} z_{t}(x, t) \frac{\sqrt{1-k^{2} x^{2}}}{\sqrt{1+k t}} z_{x}(x, t) \frac{k q(x)}{\sqrt{1-k^{2} x^{2}}} d x d t\right| \\
& \quad \leq \frac{\varepsilon}{2} \int_{0}^{T} \int_{0}^{1} \alpha_{k}(t)\left|z_{t}(x, t)\right|^{2} d x d t+\frac{1}{2 \varepsilon} \int_{0}^{T} \int_{0}^{1} \beta_{k}(x, t)\left|z_{x}(x, t)\right|^{2} g(x) d x d t \\
& \quad \leq \frac{\varepsilon}{2} \int_{0}^{T} \int_{0}^{1} \alpha_{k}(t)\left|z_{t}(x, t)\right|^{2} d x d t+\frac{1}{2 \varepsilon} k^{2} M^{2} \int_{0}^{T} \int_{0}^{1} \beta_{k}(x, t)\left|z_{x}(x, t)\right|^{2} d x d t .
\end{aligned}
$$

Take $\varepsilon=k M<1$, then it is easy to check that

$$
\varepsilon=k M=\frac{k^{2} M^{2}}{\varepsilon}<1
$$

By the value of $M$, we have

$$
k \frac{1}{2 k} \ln \frac{1+k}{1-k}<1
$$

From this, it follows that

$$
k<1-\frac{2}{1+e^{2}} .
$$

Then when

$$
k \in\left(0,1-\frac{2}{1+e^{2}}\right)
$$

we obtain

$$
\left|\int_{0}^{T} \int_{0}^{1} k p(x) z_{t}(x, t) z_{x}(x, t) d x d t\right| \leq k M \int_{0}^{T} E(t) d t .
$$

Hence, by (4.7)-(4.9), we derive

$$
\begin{gathered}
-\frac{1}{2} q(0) \int_{0}^{T} \beta_{k}(0, t)\left|z_{x}(0, t)\right|^{2} d t \\
\geq(1-k M) \int_{0}^{T} E(t) d t-\frac{2 M}{1-k} E_{0} \\
\quad=\left[\frac{1-k M}{k} \ln (1+k T)-\frac{2 M}{1-k}\right] E_{0} .
\end{gathered}
$$


Let

$$
T_{k}^{*}=\frac{e^{\frac{2 k M}{(1-k)(1-k M)}}-1}{k}
$$

If $T>T_{k}^{*}$, we have $\frac{1-k M}{k} \ln (1+k T)-\frac{2 M}{1-k}>0$. Also,

$$
\begin{aligned}
& -\frac{1}{2} q(0) \int_{0}^{T} \beta_{k}(0, t)\left|z_{x}(0, t)\right|^{2} d t \\
& \quad \geq C\left[\frac{1-k M}{k} \ln (1+k T)-\frac{2 M}{1-k}\right]\left(\left|z^{0}\right|_{H_{0}^{1}(0,1)}^{2}+\left|z^{1}\right|_{L^{2}(0,1)}^{2}\right) .
\end{aligned}
$$

From this we get (2.7). This completes the proof of Theorem 2.4.

Remark 4.2 It is easy to check that

$$
\begin{aligned}
T_{0}^{*} & \triangleq \lim _{k \rightarrow 0} T_{k}^{*}=\lim _{k \rightarrow 0} \frac{e^{\frac{2 k M}{(1-k)(1-k M)}}-1}{k}=\lim _{k \rightarrow 0} \frac{\frac{2 k M}{(1-k)(1-k M)}}{k} \\
& =\lim _{k \rightarrow 0} 2 M=\lim _{k \rightarrow 0} 2 \frac{1}{2 k} \ln \frac{1+k}{1-k}=\lim _{k \rightarrow 0} \frac{\ln \frac{1+k}{1-k}}{k}=2 .
\end{aligned}
$$

It is well known that the wave equation (1.2) in the cylindrical domain is null controllable at any time $T>T_{0}^{*}$. However, we do not know whether the controllability time $T_{k}^{*}$ is sharp.

\section{Competing interests}

The authors declare that they have no competing interests.

\section{Authors' contributions}

All results belong to $C L$ and SL. All authors read and approved the final manuscript.

\section{Author details}

${ }^{1}$ College of Applied Mathematics, Jilin University of Finance and Economics, Changchun, 130117, China. ${ }^{2}$ School of Mathematics and Statistics, Northeast Normal University, Changchun, 130024, China. ${ }^{3}$ Educational Administration, Jilin University of Finance and Economics, Changchun, 130117, China.

\section{Acknowledgements}

This work is supported by the National Science Foundation of China 11171060, 11371084 and Department of Education Program of Jilin Province under grants 2012187 and 2013287. Moreover the authors are grateful to anonymous referees for their constructive comments and suggestions, which led to improvement of the original manuscript. The authors are grateful to Christopher D. Rualizo for patient and meticulous work.

Received: 24 August 2013 Accepted: 17 February 2014 Published: 24 Feb 2014

\section{References}

1. Milla Miranda, M: Exact controllability for the wave equation in domains with variable boundary. Rev. Mat. Univ. Complut. Madr. 9, 435-457 (1996)

2. Bardos, C, Chen, G: Control and stabilization for the wave equation. Part III: domain with moving boundary. SIAM J. Control Optim. 19, 123-138 (1981)

3. Araruna, FD, Antunes, GO, Medeiros, LA: Exact controllability for the semilinear string equation in the non cylindrical domains. Control Cybern. 33, 237-257 (2004)

4. Cui, L, Liu, X, Gao, H: Exact controllability for a one-dimensional wave equation in non-cylindrical domains. J. Math. Anal. Appl. 402, 612-625 (2013)

5. Milla Miranda, M: HUM and the wave equation with variable coefficients. Asymptot. Anal. 11, $317-341$ (1995)

10.1186/1687-2770-2014-47

Cite this article as: Cui and Song: Exact controllability for a wave equation with fixed boundary control. Boundary Value Problems 2014, 2014:47 